\title{
Deformation and Clusterization in Atomic Nuclei
}

\author{
J. Cseh*, A. Algora*, J. Darai ${ }^{\dagger}$, P. O. Hess**, N. V. Antonenko ${ }^{\ddagger}$, R. V. Jolos ${ }^{\ddagger}$ \\ and W. Scheid \\ *Institute of Nuclear Research, Debrecen, Hungary \\ ${ }^{\dagger}$ Institute of Experimental Physics, University of Debrecen, Debrecen, Hungary \\ ${ }^{* *}$ Instituto de Ciencias Nucleares, UNAM, Mexico \\ ${ }^{\ddagger}$ Institut für Theoretische Physik der Justus-Liebig-Universität, Giessen, Germany
}

\begin{abstract}
The relation of the quadrupole deformation and clusterization in atomic nuclei is studied from the viewponts of energetics and exclusion principle.
\end{abstract}

Keywords: quadruploe deformation and clusterization, SU(3) symmetry, selection rule, energetic stability

PACS: $21.60 . \mathrm{Fw}, 21.60 . \mathrm{Gx}$.

As for the quadrupole deformation of the atomic nuclei is concerned, today we know not only normal deformed states, but superdeformed and hyperdeformed states as well. As for the clusterization of the atomic nucleus is concerned, today we know not only alpha-clusters, but exotic clusters (e.g. ${ }^{14} \mathrm{C}$ ) and very exotic clusters (e.g. ${ }^{108} \mathrm{Mo}$ ) as well. Therefore, the classical question: how these two phenomena, i.e. quadrupole deformation and clusterization are related to each other, is more interesting than ever before.

One of the ways of studying this interrelation is provided by the application of cluster models. Indeed, several aspects of the problem has been investigated by applying cluster models to both light and heavy nuclei.

A simple and successful cluster model is the local potential model [1] in which a local potential describes the cluster-cluster interaction. In many cases this model is able to reproduce the energies of the cluster states, their half-lives, and electromagnetic transitions. The cluster-configuration is not determined in this model directly (it is an a priori assumption), therefore, external arguments have to be applied for their determination. Several such arguments have been tested, the most general one is based on the binding energies. According to that the larger

$$
D(1,2)=\left[B(1)-B_{L}(1)\right]+\left[B(2)-B_{L}(2)\right]
$$

value corresponds to more probable clusterization. Here $B(i)$ is the experimental binding energy of the ith cluster and $B_{L}(i)$ stands for the liquid drop value [2]. In a more refined version of the method this argument of the energetic stability is combined with another empirical rule of the no-dipole constraint:

$$
\frac{Z_{1}}{A_{1}} \approx \frac{Z_{2}}{A_{2}} \approx \frac{Z_{T}}{A_{T}},
$$

where $Z$ and $A$ stand for charge and mass number, respectively, 1 and 2 indicate the clusters, while $T$ means total. 
Another model which applies also a potential for the description of the intercluster interaction is the dinuclear system model (DNS) [3]. This approach is able to take into account different cluster-configurations, characterised by the charge and mass-asymmetry coordinates, which are taken as a dynamical variables, thus their values are determined by a differential equation. In this way the best possible configuration is provided by the internal arguments of the model, based also on the best energetic circumstances, but in this case the cluster-cluster interaction (both nuclear and Coulombic) are included as well.

When we consider the problem of the building up of an atomic nucleus from nucleons (also when it is done in a two-step procedure, by applying clusters of nucleons as building blocks), there are two basic principles which govern the process: the energyminimum principle, and the Pauli-exclusion principle. The first one is taken care by the phenomenologic potential models mentioned above, the second one, however, is not. It can be dealt only microscopically, and it is usually a very complicated problem. In order to illustrate the difficulties, let us take the simple example of the ${ }^{18} \mathrm{O}$ nucleus.

When ${ }^{18} \mathrm{O}$ is described in the shell model, it can be considered as two neutrons in the average potential of the ${ }^{16} \mathrm{O}$ core, therefore, it is a two-body-problem in a limited model space. (Even in a better approximation, when particle-hole excitations are allowed the problem is still tractable.) When, however, we describe it in terms of a microscopic cluster model, the antisymmetrization of the 18-body wave-functions has to be carried out. The problem is even more difficult, of course, if instead of 18 nucleons we have $A=118$ or $A=218$. Then the fully microscopic description is out of question.

How can we take into account the consequences of the exclusion principle, when the number of nucleons is large? Like in many other problems in physics it turns out that symmetry-considerations can provide us with a simple solution to a complex problem. The logic of the solution is the following: if we have a symmetry, then a conservation law follows from it, providing us with conserved quantities, i.e. with good quantum numbers. Based on them one can formulate a selection rule, which helps us to distinguish between Pauli-allowed and Pauli-forbidden states.

The $U(3)$ symmetry is known to be a good approximative symmetry in nuclei [4]. Based on this symmetry one can formulate a simple selection rule (for binary clusterization) [5]:

$$
\left[n_{1}, n_{2}, n_{3}\right]=\left[n_{1}^{(1)}, n_{2}^{(1)}, n_{3}^{(1)}\right] \otimes\left[n_{1}^{(2)}, n_{2}^{(2)}, n_{3}^{(2)}\right] \otimes\left[n^{R}, 0,0\right] .
$$

Here $\left[n_{1}, n_{2}, n_{3}\right]$ is the set of good quantum numbers (labels of an irreducible representation) characterising a specific state (e.g. the ground state) of the parent nucleus, the superscript 1 or 2 refer to the clusters, while $R$ stands for their relative motion. This selection rule was derived first for harmonic oscillator interactions [6,7], but it turns out to be valid for more general interactions as well [8]. The selection rule says that if one of the set of $U(3)$ quantum numbers obtained from the triple product of he right-hand side matches with that of the left-hand side, then the cluster-configuration is Pauli-allowed, if not it is forbidden.

When a cluster configuration is forbidden, we can characterise its forbiddenness quantitatively in the following way [9]. The distance between a $U(3)$ reaction channel and the irrep of the parent nucleus is defined as: $\min \left(\sqrt{\left(\Delta n_{1}\right)^{2}+\left(\Delta n_{2}\right)^{2}+\left(\Delta n_{3}\right)^{2}}\right)$, where 
$\Delta n_{i}=\left|n_{i}-n_{i, k}^{c}\right|$. Here $n_{i}$ refers to the $U(3)$ representation of the parent nucleus, while $n_{i, k}^{c}$ stands for the $U(3)$ representation of channel $c$, obtained from the right-hand-side of Eq.(3), while the $k$ index distinguishing the different product-representations. Based on this quantity we determine, for reasons of convenience, the reciprocal forbiddenness $S$ in such a way, that $0 \leq S \leq 1$ :

$$
S=\frac{1}{1+\min \left(\sqrt{\left(\Delta n_{1}\right)^{2}+\left(\Delta n_{2}\right)^{2}+\left(\Delta n_{3}\right)^{2}}\right)} .
$$

Then $S=0$, and $S=1$ correspond to completely forbidden and allowed clusterizations, respectively.

It is worth stressing here that the conditions formulated by Eqs (1-4) for the selection of clusterizations (both from the energetics and from the exclusion principle point of views) can be generalised to ternary and multicluster configurations in a straighforward way.

The application of the present considerations to the binary clusteriztions of the ${ }^{36} \mathrm{Ar}$ nucleus give the result as shown in Fig. 1. The superdeformed sate of this nucleus has been identified experimentally [10], while for the hyperdeformed state there is a theoretical prediction [11]. A more detailed discussion on the application of the microscopic selection rule to this system is given in [12]. In short we can summarise its result by saying that in the normal deformed state the asymmetric clusterization is preferred, in the hyperdeformed state the symmetric clusterization is allowed, while the situation of the superdeformed state is in between: there is an island of allowed clusterconfigurations which does not include the most symmetric, and the most asymmetric fragmentations. Our preliminary results [13] for the binary clusterizations of the ${ }^{40} \mathrm{Ca}$ nucleus show some similar features.

Concerning the energetic stability of the binary cluster-configurations, it is remarkable, that the simple binding energy argument and the more detailed DNS model calculation results in the same sequence of clusterizations: alpha $>$ berilium $>$ carbon $>$ oxigen. (Preliminary calculations show that for ${ }^{40} \mathrm{Ca}$ the carbon is energetically more favoured than the berilium, from both models.)

In conclusion we can say that the two aspects of nucler clusterization, i.e. the energetic preference and the exclusion principle are complementary to each other, therefore, they do not necessarily prefer the same cluster-configuration. The likely candidates are those ones, which are favoured by both aspects. Another interesting finding of our calculations is that the same cluster-configuration (e.g. ${ }^{24} \mathrm{Mg}+{ }^{12} \mathrm{C}$ ) can be present both in the ground state and in the superdeformed as well as in the hyperdeformed state of a nucleus $\left({ }^{36} \mathrm{Ar}\right)$, if we do not apply oversimplifying model-assumptions, i.e. fully appreciate the deformed nature of the clusters, their possible relative orientations with respect to he molecular axis, and the exclusion principle.

For heavy nuclei the $U(3)$ symmetry is not valid in its original form, as we discussed here, however, a generalised version of it, called effective or quasidynamical $U(3)$ symmetry may still survive. Based on that concept the present considerations can be extended to heavy nuclei as well [14].

Work is in progress for the description of ternary cluster-configurations in a similar manner [15]. 

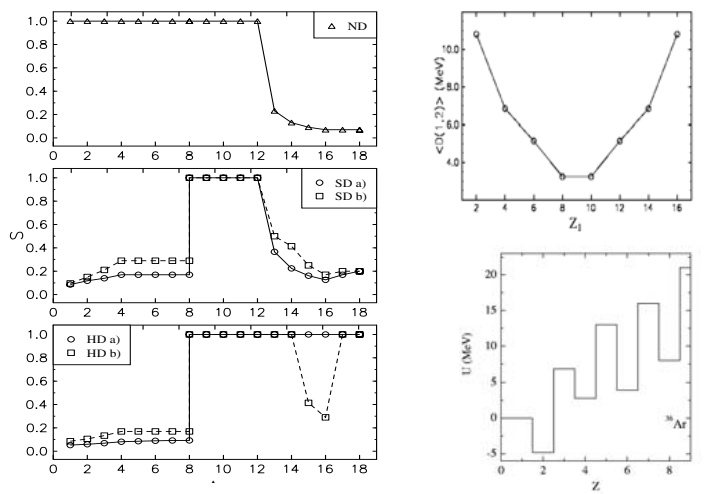

FIGURE 1. Left panel: reciproc forbiddenness (S) for the ${ }^{36} \mathrm{Ar}$ clusterizations. ND stands for the normal deformed (ground) state, while SD and HD indicate super and hyperdeformed states, respectively. a) and b) correspond to two candidates for the $U(3)$ symmetries of the SD and HD states. Right panel: Energetic stability of binary clusterizations in ${ }^{36} \mathrm{Ar}$, based on binding energies (upper part) and from DNS modelcalculation (lower part). $\mathrm{D}(1,2)$ is defined in Eq. (1), while U stands for potential energy.

This work was supported by the OTKA (Grant Nos. T37502, T46791), the MTA-CONACyT joint project and by DGAPA (IN119002). J. Cseh recognizes partial support of the Alexander von Humboldt foundation, while A. Algora that of the János Bolyai research fellowship.

\section{REFERENCES}

1. B. Buck, A. C. Merchant, S. M. Perez, Phys. Rev. Lett. 76 (1996) 380, and references therein.

2. B. Buck, A. C. Merchant, and S. M. Perez, Few-Body Systems 29 (2000) 53;

B. Buck, A. C. Merchant, M. J. Horner, and S. M. Perez, Phys. Rev. C61 (2000) 024314.

3. V.V. Volkov, Phys. Rep. 44, 93 (1978); Deep inelastic nuclei reactions (Energoizdat, Moscow, 1982);

W. Greiner, J.Y. Park, and W. Scheid, Nuclear Molecules(World Scientific, Singapore, 1995); G.G.

Adamian et al., Phys. Rev. C67 (2003) 054303; C69 (2004) 054310; Acta Phys. Pol. B34 (2003) 2147.

4. J. P. Elliot, Proc. R. Soc. A 245 (1958) 128, 562.

5. J. Cseh, Phys. Lett. 281B (1992) 173;

J. Cseh and W. Scheid J. Phys. G18 (1992) 1419;

J. Cseh, J. Phys. G19 (1993) L97;

J. Cseh and G. Lévai, Ann. Phys. (N.Y.) 230 (1994) 165.

6. K. Wildermuth and Th. Kanellopoulos, Nucl. Phys. 7 (1958) 150.

7. B. F. Bayman and A. Bohr, Nucl. Phys. 9 (1958/59) 596.

8. J. Cseh, G. Lévai, A. Algora, P.O. Hess, A. Intasorn, and K. Katō, Heavy Ion Phys. 12 (2000) 119.

9. A. Algora and J. Cseh, J. Phys. G: Nucl. Part. Phys.22 (1996) L39.

10. C. E. Svensson, et. al., Phys. Rev. Lett. 85 (2000) 2693;

C. E. Svensson, et. al., Phys. Rev. C63 (2001) 061301(R).

11. W. D. M. Rae and A. C. Merchant, Phys. Lett. B279 (1992) 207.

12. J. Cseh, A. Algora, J. Darai, P. O. Hess, Phys. Rev. C70 (2004) 034311.

13. J. Darai, J. Cseh, A. Algora, N. V. Antonenko, R. V. Jolos, and W. Scheid, in preparation.

14. A. Algora, contribution to this conference.

15. A. Algora, J. Cseh, J. Darai, P. O. Hess, in preparation. 
Copyright of AIP Conference Proceedings is the property of American Institute of Physics. The copyright in an individual article may be maintained by the author in certain cases. Content may not be copied or emailed to multiple sites or posted to a listserv without the copyright holder's express written permission. However, users may print, download, or email articles for individual use. 